\title{
Management of Multicentric Myopericytoma in the Maxillofacial Region: A Case Report
}

\author{
Fanglong $\mathrm{Wu}^{\mathrm{a}}$ Jun Sun ${ }^{\mathrm{a}}$ Jiazeng Dong ${ }^{\mathrm{a}, \mathrm{c}} \quad$ Xiaoyi Wang ${ }^{\mathrm{a}, \mathrm{b}}$ \\ Qinghong $\mathrm{Gao}^{\mathrm{a}}$, b \\ ${ }^{a}$ State Key Laboratory of Oral Diseases, and ${ }^{b}$ Department of Oral and Maxillofacial \\ Surgery, West China Hospital of Stomatology, Sichuan University, Chengdu, and \\ ${ }^{\mathrm{C}}$ Department of Dentistry, The Second Renmin Hospital of Jiaxing, Jiaxing, China
}

\author{
Key Words \\ Complication · Management · Myopericytoma
}

\begin{abstract}
Myopericytoma (MPC) is a rare kind of benign neoplasm, showing derivation from perivascular myoid cells. About 115 cases have been reported in the English literature; however, most of the literature focuses on the description and classification of pathology. The case presented here is that of a 42-year-old woman with a surgical management experience of multicentric MPC in the maxillofacial region. Although small MPC can be completely and easily excised, large MPC, especially in certain anatomic sites, necessitates careful preoperative preparation.
\end{abstract}

\section{Introduction}

Myopericytoma (MPC), showing derivation from perivascular myoid cells, is a kind of benign neoplasm that is often misdiagnosed as sarcoma [1]. Its classification and terminology has been confusing. In 1942, hemangiopericytoma (HPC) was described as a tumor characteristically consisting of plump, spindle-shaped cells, and showing irregular, thinwalled, branched 'stag horn' blood vessels [2]. In 1996, it was proposed that cutaneous adult myofibroma overlaps with HPC, which is comprised of cells with morphological and immunohistochemical features of immature pericytes or myopericytes, so the term 'MPC' was suggested instead of myofibroma [3]. Later, three HPC-like tumor features were 
comprehensively analyzed. These pattern types were that of myofibromatosis in adults, glomangiopericytoma and MPC; these three tumors overlap in clinicopathological features and it was believed that they probably derived from (or differentiated toward) perivascular myoid cells [4]. In 2002, the World Health Organization categorized MPC as myopericytic tumors within HPC-like neoplasms [5, 6]. However, it was not until 2006 that Mentzel et al. [6] distinguished the diagnosis of MPC from differential diagnoses of other neoplasms such as angioleiomyoma and myofibroma. They precisely demonstrated that MPC represents a distinctive perivascular, myoid neoplasm of the skin and soft tissues, and that its main histological feature is the presence of numerous thin-walled blood vessels, with concentric periluminal proliferation of ovoid, plump, spindled and/or round myoid tumor cells, showing a broad morphological spectrum. On the other hand, immunohistochemical staining is useful for the diagnosis, and MPC is characteristically immunoreactive to SMA, MSA and hcaldesmon, while desmin is usually negative $[1,4,6]$.

MPC has a propensity to occur in the dermis and subcutaneous regions of the distal extremities or the head and neck region in middle-aged adults. About 115 cases have been reported; however, most of the literature focuses on the description and classification of pathology. The aim of this case report is to present a multicentric case in the maxillofacial region, including clinical and radiological features, and treatment experience. This study was approved by the Ethics Committee of the West China Hospital of Stomatology, Sichuan University.

\section{Case Report}

The patient was a 42-year-old Chinese woman who presented with a 10-year history of slowly growing masses in the maxillofacial region. In 1997, the patient found a mass in the back region of her right ear, and the diagnosis based on a biopsy of the mass was merely a benign tumor. Shortly after the excision, another mass emerged and gradually enlarged below the right earlobe region. The patient refused tumor excision due to fear of the surgery.

Since the patient was referred to the Department of Head and Neck Oncology, West China Hospital of Stomatology, Sichuan University, in 2007, she had been feeling slight pain in the mass of the right parotid gland. Physical examination revealed an approximately $6 \times 5$ $\mathrm{cm}$ mass in the region of the right parotid gland. It was a multinodular lesion without mobility and tenderness, and positive to the position changing enlargement test. There was no evidence of sensory deficit or facial palsy. On panoramic radiography, there was a smallsized deformity in the right ramus. The computed tomography (CT) scan demonstrated multicentric soft tissue masses in the region of the right parotid gland, cheek, infratemporal fossa and parapharyngeal spaces; these masses enhanced peripherally with central heterogeneous irregular nonenhancement after administration of a contrast medium (fig. 1).

To reduce blood loss during surgery, digital subtraction angiography (DSA) and superselective embolization were performed prior to the operation. DSA disclosed soft tissue in the right parotid gland region, with an abundant blood supply from branches of the right external carotid artery. With a guide wire, a $5 \mathrm{~F}$ sheath was used to superselectively catheterize the subbranches of the right external carotid artery and embolize them with 550-750 um PVA particles. Immediate postinjection angiography showed an occlusion of the subbranches to the focus (fig. 2).

Under general anesthesia, the mass in the right parotid gland region was completely excised without much blood loss. It was light yellow and about $6 \times 5 \times 5 \mathrm{~cm}$. In attempting to remove other masses in the parapharyngeal space, the authors removed the right ramus and 
tried to pass through the lateral and medial pterygoid muscles. Unfortunately, there was a lot of blood loss from the pterygoid venous plexus and, at that time, the total blood loss had reached about 1,600 ml. The authors had to give up the removal of the other masses in the parapharyngeal space. The patient had an uneventful recovery, except for right facial palsy, and was discharged 7 days following the operation.

The lesion was identified as MPC. The pathological findings showed numerous gaping and branching, and thin-walled vessels concentrically surrounded by spindle ovoid myoid tumor cells with eosinophilic cytoplasm (fig. 3). Hyalinization was present, but there was no necrosis, nuclear anaplasia, increased mitoses or infiltrative growth. There was positive immunoreactivity for SMA, MSA and desmin, but CD34 and CD99 staining were negative.

The patient had no more pain and was reviewed at 3- to 6-month intervals. There was no sign of recurrence in the right parotid gland region, and there was no obvious enlargement in the residual lesions 5 years postoperatively. She has had regular follow-up.

\section{Discussion}

A review of the English literature was done in PubMed - the search was conducted with the word 'myopericytoma' for literature dating from 1998 to 2012 - and revealed 115 cases of MPC mainly from 5 papers and other case reports [1, 4-8]. MPC was slightly male predominant: the male to female ratio was 1.25:1. The patients' ages ranged from 10 to 87 years, with a median age of 49 years. Eight patients were children, while 85 patients were over 40 years of age. The most common location was the lower extremities, followed by the upper extremities, head and neck, and trunk, including the spine, kidney, lung and pancreatic gland. In the oral maxillofacial region, MPC usually presented in the neck, parotid gland, nose, infraorbital region, lip, cheek and tongue. In most cases, the neoplasms were superficial; there were only a few cases of neoplasms large enough to reach deep soft tissues. Several cases were painful. In a few rare cases, the lesions were multicentric and multiple anatomic regions were involved. The present case was quite rare in this sense.

Most lesions present as well-circumscribed, blue-grey colored, single or multiple superficial nodules and are detectable by ultrasonography. But for deep lesions, CT scan and magnetic resonance imaging (MRI) are better methods. Routine CT scans can demonstrate the mass, with the peripheral portion of the mass markedly enhanced, but the central portion might remain poorly enhanced even after injection of a contrast medium [9]. It was the same with the CT images in the present case. On MRI, the mass may appear hypo- to isointense on T1-weighted sequences and hyperintense on T2-weighted sequences. There was an intense homogeneous contrast enhancement after administration of a contrast medium $[7,10]$. These images are helpful during surgery.

Since MPC is usually a benign tumor, one can expect good results after complete surgical excision, with local recurrence only in very rare cases [6]. Sometimes it is difficult to distinguish a local recurrence from a residual nodule, especially in multicentric cases. There were some residual nodules in the present case; however, the patient refused to undergo further surgery in order to avoid more discomfort. In complicated cases like this one, complete excision is difficult. In this case, there were several complications: (1) blood loss: despite preoperative DSA and embolization, and no obvious bleeding in the process of removing the parotid gland region mass, there was a lot of blood loss from the pterygoid venous plexus, and (2) facial palsy: in the operation, dissection of the facial nerve was performed very carefully, and the branches were preserved. Facial palsy might have been due to nerve trauma during tumor removal, which, unfortunately, did not recover. For these 


\begin{tabular}{l|l}
\hline DOI: $10.1159 / 000353625$ & $\begin{array}{l}\text { C } 2013 \text { S. Karger AG, Basel } \\
\text { www.karger.com/cro }\end{array}$ \\
\hline
\end{tabular}

Wu et al.: Management of Multicentric Myopericytoma in the Maxillofacial Region: A Case Report

two reasons, treatment in this case was not considered a success, although the patient was satisfied with the outcome.

\section{Conclusion}

MPC in the oral and maxillofacial region is rare, and its diagnostic efficacy depends on histopathological features, especially immunohistochemical confirmation. Although small MPC can be completely excised easily, large ones, especially in certain anatomic sites, necessitate careful preoperative preparation. CT scans or MRI and DSA are helpful for surgical management.

\section{Acknowledgements}

The authors are very grateful to the patient for permission to use the photographs. The authors are also very grateful to Dr. Yu Chen, Head of the Department of Histopathology, West China Hospital of Stomatology, Sichuan University, for her generous contribution of case material to this study.

\section{Disclosure Statement}

There is no conflict of interest.

\section{References}

1 Dray MS, McCarthy SW, Palmer AA, Bonar SF, Stalley PD, Marjoniemi V, Millar E, Scolyer RA: Myopericytoma: a unifying term for a spectrum of tumours that show overlapping features with myofibroma. A review of 14 cases. J Clin Pathol 2006;59:67-73.

2 Stout AP, Murray MR: Hemangiopericytoma: a vascular tumour featuring Zimmerman's pericytes. Ann Surg 1942;116:26-33.

-3 Requena L, Kutzner H, Hügel H, Rütten A, Furio V: Cutaneous adult myofibroma: a vascular neoplasm. J Cutan Pathol 1996;23:445-457.

4 Granter SR, Badizadegan K, Fletcher CD: Myofibromatosis in adults, glomangiopericytoma, and myopericytoma: a spectrum of tumors showing perivascular myoid differentiation. Am J Surg Pathol 1998;22:513-525.

-5 Ide F, Mishima K, Yamada H, Saito I, Horie N, Shimoyama T, Kusama K: Perivascular myoid tumors of the oral region: a clinicopathologic re-evaluation of 35 cases. J Oral Pathol Med 2008;37:43-49.

6 Mentzel T, Dei Tos AP, Sapi Z, Kutzner H: Myopericytoma of skin and soft tissues: clinicopathologic and immunohistochemical study of 54 cases. Am J Surg Pathol 2006;30:104-113.

7 Rousseau A, Kujas M, van Effenterre R, Boch AL, Carpentier A, Leroy JP, Poirier J: Primary intracranial myopericytoma: report of three cases and review of the literature. Neuropathol Appl Neurobiol 2005;31:641-648.

-8 Díaz-Flores L, Gutiérrez R, García MP, Alvarez-Argüelles H, Díaz-Flores L Jr, Madrid JF: Myopericytoma and arterial intimal thickening: the relationship between myopericytes and myointimal cells. J Cutan Pathol 2011;38:857-864.

-9 Cao J, Xu J, Li Y, Lai J, Li Q: Pulmonary myopericytoma: a case report and review of the literatures. Chin Med J 2009;122:755-757.

10 Kuczkowski J, Rzepko R, Szurowska E: Myopericytoma of the parotid gland - a pathological conundrum. J Craniomaxillofac Surg 2010;38:595-596. 

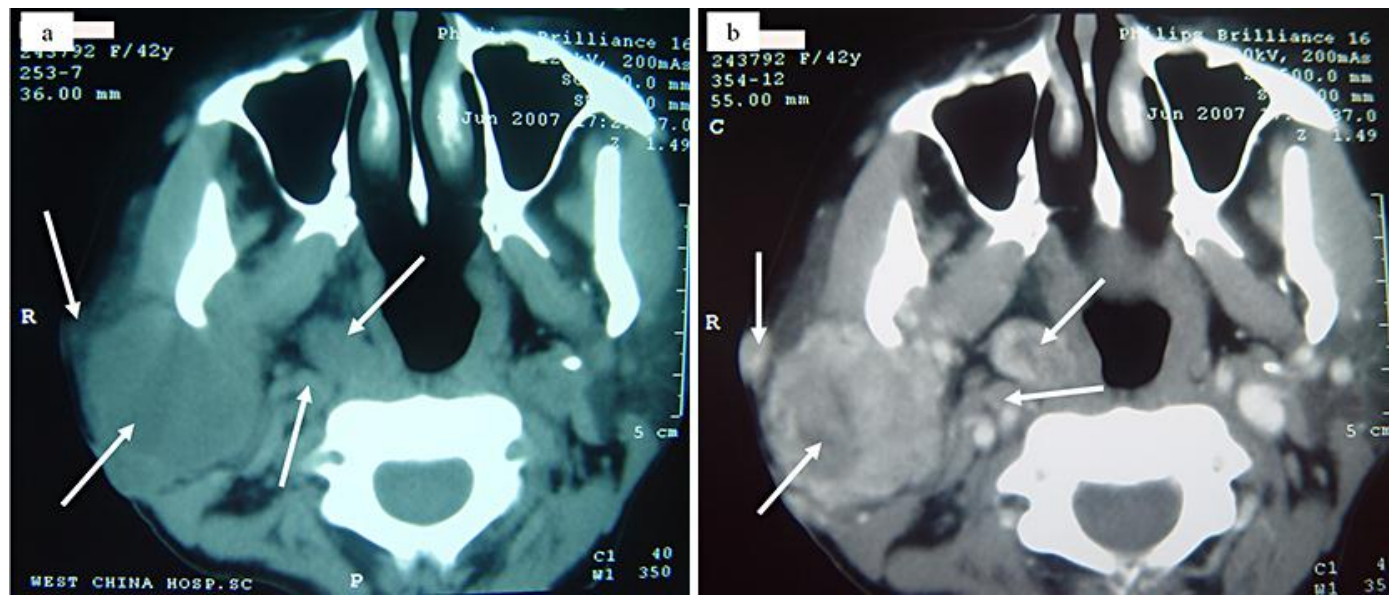

Fig. 1. CT scan. Multicentric soft tissue masses can be seen in the region of the right parotid gland and in the right parapharyngeal region. There was a small-sized deformity in the right ramus. a Before administration of a contrast medium. $\mathbf{b}$ After administration of a contrast medium. Arrows point to the tumor masses.
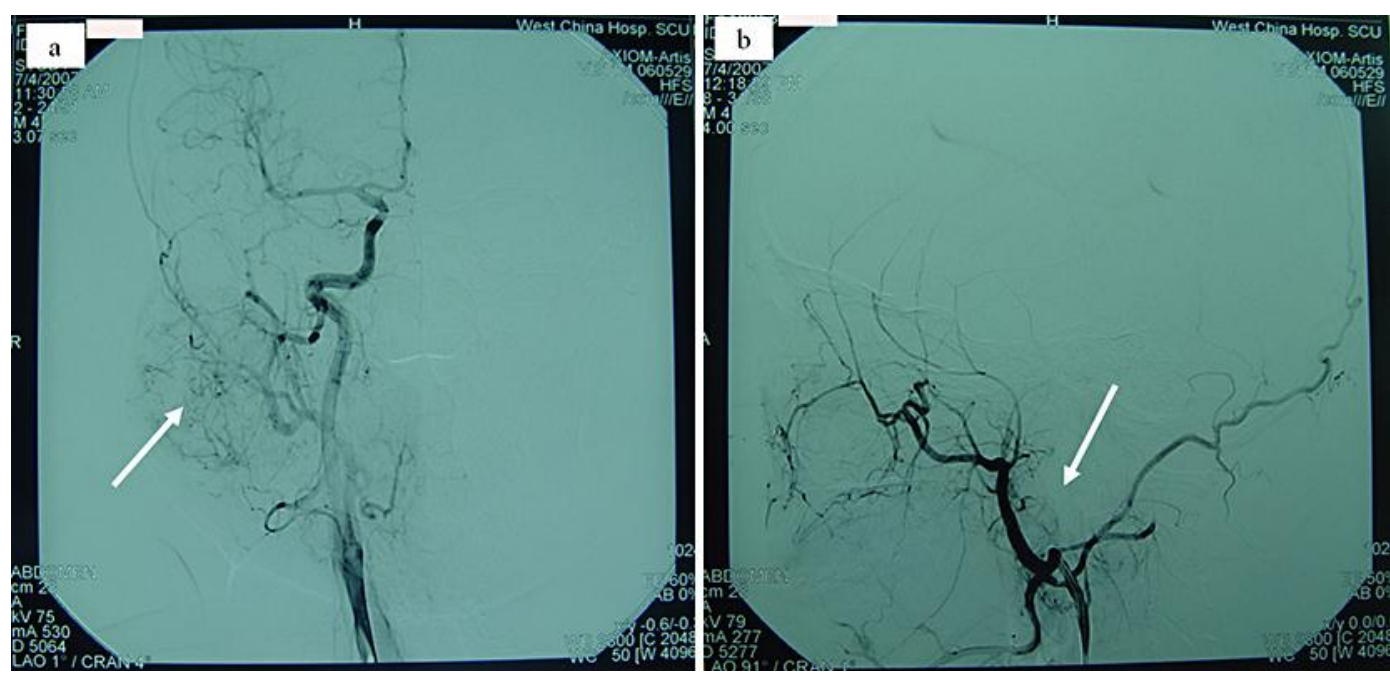

Fig. 2. DSA. a Soft tissue mass in the right parotid gland, with a rich blood supply from branches of the right external carotid artery. The arrow indicates the abnormal blood vessels. b Immediate postinjection angiography shows no visualization of the focus. The arrow indicates the disappearance of the abnormal blood vessels. 


\section{Case Reports in Oncology}

Wu et al.: Management of Multicentric Myopericytoma in the Maxillofacial Region: A Case Report



Fig. 3. Histopathological section. Numerous branching, thin-walled vessels concentrically surrounded by spindle ovoid myoid tumor cells with eosinophilic cytoplasm. HE. $\times 100$. 